\title{
Association between major patterns of dietary intake and weight status in adolescents
}

\author{
Gretchen J. Cutler ${ }^{1 *}$, Andrew Flood ${ }^{2}$, Peter J. Hannan ${ }^{2}$, Joanne L. Slavin ${ }^{3}$ \\ and Dianne Neumark-Sztainer ${ }^{2}$ \\ ${ }^{1}$ Medica Research Institute, 401 Carlson Parkway, Minnetonka, MN 55305, USA \\ ${ }^{2}$ Division of Epidemiology and Community Health, School of Public Health, University of Minnesota, Minneapolis, \\ MN 55455, USA \\ ${ }^{3}$ Department of Food Science and Nutrition, University of Minnesota, St Paul, MN, USA \\ (Submitted 2 February 2011 - Final revision received 6 September 2011 - Accepted 6 September 2011 - First published online 13 October 2011)
}

\section{Abstract}

The relationship between dietary intake and obesity is complex, and dietary pattern analysis may offer new insight. We examined associations between dietary patterns identified in a diverse cohort of adolescents and weight status cross-sectionally and over a 5-year period. Project EAT (Eating Among Teens) (Time 1) collected data on 4746 middle (younger cohort) and high school (older cohort) students in 1998-9. EAT-II (Time 2) resurveyed 2516 of the original cohort in 2003-4. All analyses were run separately by age cohort and sex. The relationship between dietary patterns identified previously (vegetable, fruit, vegetable \& fruit, starchy food, sweet \& salty snack food, and fast food) and weight status was examined using logistic regression. All analyses were adjusted for socioeconomic status, race/ethnicity and activity level (longitudinal analyses were also adjusted for baseline weight status). In crosssectional analyses, higher adherence to dietary patterns loading heavily on vegetables was associated with lower risk of overweight/ obese weight status in older and younger girls, whereas higher adherence to a 'sweet \& salty snack food' pattern was associated with lower risk in older and younger boys. These associations were found prospectively in older boys and girls, but were no longer significant in analyses adjusting for baseline weight status. We did not find consistent or intuitive associations between dietary patterns and weight status. Identified patterns may not capture the elements of diet that are truly important in determining adolescent weight, or diet may not be the primary driver in determining weight status at this age. Methodological difficulties in assessing diet must also be taken into consideration.

Key words: Dietary patterns: Factor analysis: Adolescents: Weight status

The prevalence of overweight and obesity has increased dramatically in children and adolescents in the past three decades $^{(1)}$. More research is needed to determine how dietary factors are related to obesity in youth ${ }^{(2)}$. Research up to this point has not shown a consistent association between most dietary factors and obesity in children and adolescents ${ }^{(2)}$. This may be partly due to the traditional method used in nutritional epidemiology. The majority of studies examining the relationship between dietary intake and obesity in youth have focused on intake of individual nutrients, foods or food groups. Although these studies are informative, and more research is certainly warranted, a new approach is needed if we want to further understand the diet-obesity relationship in youth. Dietary pattern analysis, an approach that examines the joint effects of multiple dietary components, has been used successfully in adults, and further exploration in adolescent populations is warranted ${ }^{(2,3)}$.

Dietary patterns are usually defined in two ways: a priori through the use of a diet index based on present nutrition knowledge or theory, or a posteriori through statistical methods such as principal components factor analysis or cluster analysis using dietary data collected from a specific study population $^{(4)}$. Dietary pattern analysis has emerged as an alternative to the traditional methods of examining single foods or nutrients ${ }^{(5)}$. There has been a growing appreciation that the overall pattern of dietary intake should be considered when studying relationships between nutrition and chronic conditions because of the complexity of nutrient interactions $^{(6)}$. The dietary pattern approach has intuitive appeal as the human diet does not consist of a single nutrient or

Abbreviations: EAT, Eating Among Teens; MVPA, moderate-to-vigorous physical activity; SES, socioeconomic status; YAQ, Youth/Adolescent Questionnaire.

*Corresponding author: G. J. Cutler, fax +1952 9922473, email gretchen.cutler@medica.com 
food, but instead represents a complex set of highly correlated dietary exposures ${ }^{(7,8)}$.

The dietary pattern approach has rarely been used in adolescent populations, particularly with regard to studying the relationship between dietary intake and obesity. Only five studies have examined the associations between dietary patterns and obesity in adolescents ${ }^{(9-13)}$, and only one has taken place in the $\mathrm{USA}^{(9)}$. So far these studies do not show a clear association between dietary patterns and obesity in adolescents, although it is hard to compare results from these studies because of differences in study populations and statistical analysis methods. More well-designed prospective studies on diverse adolescent populations are needed before we can determine the usefulness of this method for examining the relationship between dietary intake and obesity in this age group $^{(3)}$.

The primary aim of the present study was to examine the relationship between adherence to dietary patterns previously identified through principal components factor analysis and weight status in adolescents ${ }^{(14)}$. The present study adds to the literature by using dietary pattern analysis on a large and ethnically and socioeconomically diverse cohort of adolescents. It was hypothesised that higher adherence to dietary patterns characterised by vegetables and fruits would be associated with a lower risk of overweight or obese weight status, and that the opposite would be true for patterns characterised by snack foods and fast food.

\section{Materials and methods}

\section{Study design and population}

Project EAT (Eating Among Teens) is an observational study of the socioenvironmental, personal and behavioural determinants of dietary intake and weight status among a large and ethnically diverse adolescent population. In Project EAT-I (Time 1), 4746 middle school and high school students in thirty-one Minnesota schools in Minneapolis, St Paul and Osseo school districts completed in-class surveys and anthropometric measures during the 1998-9 academic year. Schools were selected to ensure an ethnically and socioeconomically diverse cohort, with the goal of improving the ability to generalise findings from this cohort to other adolescents. Project EAT-II (Time 2) aimed to resurvey all original participants 5 years later (2003-4). At Time 2, 2516 participants completed mailed surveys, representing $69 \%$ of those who could be contacted and $53 \%$ of the original cohort. Study characteristics of the Project EAT cohort can be found in Table 1. The present study was conducted according to the guidelines laid down in the Declaration of Helsinki, and all procedures involving human subjects were approved by the University

Table 1. Study characteristics of the Project EAT (Eating Among Teens) cohort at Time $1^{*}$ by age group and sex

(Percentages, mean values and standard deviations)

\begin{tabular}{|c|c|c|c|c|c|c|c|c|}
\hline & \multicolumn{2}{|c|}{$\begin{array}{l}\text { Older girls } \\
(n 1253)\end{array}$} & \multicolumn{2}{|c|}{$\begin{array}{l}\text { Older boys } \\
(n 1257)\end{array}$} & \multicolumn{2}{|c|}{$\begin{array}{l}\text { Younger girls } \\
\quad(n 534)\end{array}$} & \multicolumn{2}{|c|}{$\begin{array}{c}\text { Younger boys } \\
\quad(n 528)\end{array}$} \\
\hline & Mean & SD & Mean & SD & Mean & SD & Mean & SD \\
\hline Age at Time 1 (years) & $15 \cdot 8$ & 0.8 & $15 \cdot 9$ & 0.8 & $12 \cdot 8$ & 0.8 & $12 \cdot 9$ & 0.8 \\
\hline Age at Time 2 (years) & $20 \cdot 3$ & 0.8 & 20.5 & 0.8 & $17 \cdot 2$ & 0.6 & $17 \cdot 3$ & 0.6 \\
\hline BMI $\left(\mathrm{kg} / \mathrm{m}^{2}\right)$ & $22 \cdot 6$ & $4 \cdot 3$ & $23 \cdot 0$ & $4 \cdot 3$ & $21 \cdot 3$ & 4.6 & $21 \cdot 3$ & 4.8 \\
\hline MVPA (h/week) & $5 \cdot 7$ & 4.4 & $7 \cdot 7$ & 4.8 & $5 \cdot 6$ & 4.3 & 7.4 & 4.8 \\
\hline \multicolumn{9}{|l|}{ Weight status $\dagger(\%)$} \\
\hline Underweight & \multicolumn{2}{|c|}{$3 \cdot 8$} & \multicolumn{2}{|c|}{$6 \cdot 6$} & \multicolumn{2}{|c|}{$6 \cdot 7$} & \multicolumn{2}{|c|}{$8 \cdot 9$} \\
\hline Average weight & \multicolumn{2}{|c|}{73.1} & \multicolumn{2}{|c|}{$68 \cdot 0$} & \multicolumn{2}{|c|}{$67 \cdot 2$} & \multicolumn{2}{|c|}{$60 \cdot 3$} \\
\hline Overweight & & & & & & & \multirow{2}{*}{\multicolumn{2}{|c|}{$\begin{array}{l}16 \cdot 9 \\
14 \cdot 0\end{array}$}} \\
\hline Obese & \multicolumn{2}{|c|}{7.5} & \multicolumn{2}{|c|}{$10 \cdot 3$} & \multicolumn{2}{|c|}{11.4} & & \\
\hline \multicolumn{9}{|l|}{ Race/ethnicity (\%) } \\
\hline White & \multicolumn{2}{|c|}{$57 \cdot 6$} & \multicolumn{2}{|c|}{$63 \cdot 1$} & \multicolumn{2}{|c|}{$37 \cdot 8$} & \multicolumn{2}{|c|}{38.0} \\
\hline Black & \multicolumn{2}{|c|}{$13 \cdot 6$} & \multicolumn{2}{|c|}{$12 \cdot 9$} & \multicolumn{2}{|c|}{$18 \cdot 2$} & \multicolumn{2}{|c|}{$20 \cdot 4$} \\
\hline Hispanic & \multirow{2}{*}{\multicolumn{2}{|c|}{$\begin{array}{c}3.7 \\
19.5\end{array}$}} & \multirow{2}{*}{\multicolumn{2}{|c|}{$\begin{array}{c}4.8 \\
15 \cdot 1\end{array}$}} & \multirow{2}{*}{\multicolumn{2}{|c|}{$\begin{array}{c}7.0 \\
22 \cdot 6\end{array}$}} & \multicolumn{2}{|c|}{$8 \cdot 6$} \\
\hline Asian & & & & & & & & \\
\hline Hawaiian/Pacific Islander & \multicolumn{2}{|c|}{0.3} & \multicolumn{2}{|c|}{0.6} & & & & \\
\hline Native American & & & & & & & & \\
\hline Mixed & & & & & & & & \\
\hline SES $\ddagger(\%)$ & & & & & & & & \\
\hline Low & & & & & & & & \\
\hline Middle low & & & & & & & & \\
\hline Middle & & & & & & & & \\
\hline Middle high & & & & & & & & \\
\hline High & & & & & & & & \\
\hline
\end{tabular}

MVPA, moderate-to-vigorous physical activity; SES, socioeconomic status.

* All variables from Time 1 except age at Time 2 .

† Weight status classified using Must et al. ${ }^{(16)}$ classification.

‡Prime determinant of SES was parental education level (higher level of either parent). An algorithm was developed that also took into account family eligibility for public assistance, eligibility for free or reduced-cost school meals, and employment status of mother or father ${ }^{(26)}$. 
of Minnesota's Institutional Review Board Human Subjects Committee. Written informed consent was obtained from all subjects.

\section{Weight status}

BMI was based on self-reported height and weight measures and was calculated using the formula $\mathrm{kg} / \mathrm{m}^{2}$. At Time 1, both measured and self-reported height and weight were collected, but at the 5-year follow-up (Time 2), participants completed mailed surveys and therefore only self-reported measures were collected. Thus, in the present analysis, selfreported values are used to be consistent in the measures used at both time points. Although we would have preferred to have had measured BMI at both time points, the correlations between reported and measured BMI at Time 1 were high ( $r 0.85$ for girls and $r 0.89$ for boys $)^{(15)}$, lessening our concern. For weight status, the Must et al. ${ }^{(16)}$ classification was used, because it provides continuous values from childhood to adulthood based on the first National Health and Nutrition Survey (NHANES I), and in the present analysis, adolescents were followed through late adolescence/young adulthood. Participants were classified as 'overweight/obese' if BMI values were $\geq 85$ th percentile for sex and age ${ }^{(16)}$.

\section{Dietary intake}

Dietary intake was assessed at Time 1 and Time 2 using the Youth/Adolescent Questionnaire (YAQ), a 152-item semiquantitative FFQ. At Time 1, the YAQ was administered by trained Project EAT staff in middle and high school classrooms, whereas at Time 2, the YAQ was self-administered, as it was a mailed survey. The YAQ is based on the adult Nurses' Health Study FFQ, and was developed specifically for use with adolescents. In comparison to the adult form, the YAQ includes foods eaten more commonly in youth, and excludes some food choices eaten more commonly by adults ${ }^{(17)}$. The validity and reproducibility of the YAQ have been examined in children and adolescents aged 9-18 years and have been found to be within acceptable ranges for dietary assessment tools ${ }^{(17,18)}$. The average correlation coefficient from a validation study comparing the YAQ to three $24 \mathrm{~h}$ dietary recalls was $0.54^{(17,19)}$. Some studies have suggested that there may be higher levels of measurement error in low socioeconomic status (SES) and minority populations ${ }^{(20,21)}$. Before using the YAQ for Project EAT, the questionnaire was pre-tested among a low-income, ethnically diverse middle school population and was found to be acceptable for comprehension ${ }^{(22)}$.

\section{Physical activity}

Moderate-to-vigorous physical activity (MVPA) was assessed at Time 1 and Time 2 using a modified version of the Leisure Time Exercise Questionnaire ${ }^{(23)}$. This measure has been shown to be reliable and to be significantly correlated with other measures of physical activity in adolescents ${ }^{(24)}$. Two questions were asked to assess how many hours were spent in strenuous or moderate physical activity behaviours in a usual week. The responses $(0,<0.5,0.5-2,2.5-4,4.5-6$ and $>6 \mathrm{~h} /$ week) for each activity were coded using the midpoints of each category $(0,0.3,1.3,3.3,5.3$ and $8 \mathrm{~h} /$ week), and these values were summed to create the hours-perweek variable.

\section{Socioeconomic status}

SES was based on adolescent report at Time 1. The prime determinant of SES was parental education level, defined by the higher level of educational attainment of either parent. An algorithm was developed that also took into account family eligibility for public assistance, eligibility for free or reduced-cost school meals and employment status of the mother or father ${ }^{(25)}$. A total of five categories were created (low, low middle, middle, upper middle and high).

\section{Ethnicity/race}

Ethnicity/race was based on the Time 1 survey question: 'Do you think of yourself as: (1) White; (2) Black or African America; (3) Hispanic or Latino; (4) Asian American; (5) Hawaiian or Pacific Islander; (6) American Indian or Native American'. Adolescents were given the option to choose more than one category, and those with multiple responses were coded as 'mixed/other'.

\section{Identification of dietary patterns}

Details of the dietary pattern analysis have been described previously ${ }^{(14)}$. Briefly, dietary patterns were identified separately both by cohort (older/younger) and sex (boys/girls) using principal components factor analysis based on dietary data from responses to the YAQ at Time 1 and Time 2. All analyses at Time 1 and Time 2 were first run separately for each age and sex subgroup to determine whether different dietary patterns would be identified in the subgroups. As different dietary patterns were identified in the younger and older subgroups at Time 2, all further analyses were run separately by age and sex subgroup. The YAQ provided frequency of consumption information on 152 separate line items, and we chose not to further group these items to reduce the number of subjective decisions made in determining the dietary patterns. Using an energy density approach, we divided each individual's daily frequency of consumption for each of the 152 line items by his or her total daily energy consumption, in order to adjust for energy. We then standardised the energy-adjusted frequency values to a mean of 0 and standard deviation of 1.0. Each of the standardised, energy-adjusted, frequency variables entered the principal components factor analysis (using PROC FACTOR in SAS statistical software, version 8.2, 2001; SAS, Cary, NC, USA). The factors were orthogonally rotated using the varimax procedure to facilitate interpretation. At Time 1 and Time 2, four factors (i.e. dietary patterns) were retained, based on Eigenvalues $>1$, an inspection of where the Scree plot began to flatten out and interpretability. Factor loadings \pm 0.30 were examined for each factor to identify those foods most strongly associated 
with that specific factor. For every subject we calculated factor scores for each of the retained dietary patterns by summing frequency of consumption multiplied by factor loadings across all food items.

At Time 1, four dietary patterns were identified in each of the four age and sex subgroups. We labelled these factors the 'vegetable' pattern, 'fruit' pattern, 'starchy food' pattern and 'sweet \& salty snack food' pattern after examining the foods that loaded heavily on each factor. Factor loadings for these patterns have been published previously ${ }^{(14)}$. Briefly, the 'vegetable' pattern loaded heavily on foods including zucchini, squash and eggplant, kale and greens, spinach, and carrots; the 'fruit' pattern loaded heavily on foods including oranges and grapefruit, apples and applesauce, grapes, bananas, and strawberries; the 'starchy food' pattern loaded heavily on foods including English muffins/bagels, grilled cheese, pancakes, macaroni and cheese, spaghetti with sauce, mashed potatoes, and lasagna; and the 'sweet \& salty snack food' pattern loaded heavily on foods including chocolate bars, cake, brownies, potato chips, and nachos.

The identical four dietary patterns found at Time 1 were no longer found for each age and sex subgroup at Time 2, although the patterns still remained fairly similar between subgroups. We identified the same separate 'fruit' and 'vegetable' patterns in the younger girls and boys at Time 2 as was identified at Time 1, but for the older boys and girls, the two separate patterns combined to create one 'fruit \& vegetable' pattern. In addition, a new dietary pattern we called 'fast food', characterised by foods one would be likely to eat at a fast food restaurant such as hamburgers, French fries, fried food and non-diet soda, was identified in the younger boys, and in the older boys and girls.

\section{Statistical analysis}

All analyses at Time 1 and Time 2 were run separately for each age and sex subgroup (younger girls, younger boys, older girls and older boys), as different dietary patterns were previously identified for the older and younger subgroups. Associations between factor scores for the identified dietary patterns and weight status were examined using multivariable logistic regression (Proc Logistic, SAS, version 8.2, 2001). Factor scores were categorised into quintiles. Logistic regression was used to calculate OR for being in the overweight/obese weight status category $v$. being in the normal weight status category for a one quintile increase in factor score for each dietary pattern. $95 \% \mathrm{CI}$ and $P$ values were used to interpret the statistical significance of hypothesis tests. Variables were chosen for adjustment on the basis of being related to both the independent variable (dietary pattern score) and the dependent variable (weight status). Models were first run adjusting for SES and race/ethnicity. Models were then run adjusting for MVPA in addition to SES and race/ethnicity, given that physical activity could influence both dietary intake and weight status. We did not adjust for energy intake in these models, as it was thought to be on the causal pathway between dietary intake and weight status. Finally, in longitudinal analyses, a third model was run that also adjusted for weight status at Time 1 . The outcome variable, weight status, was modelled as a dichotomous variable (normal $v$. overweight/obese). Analyses were run examining overweight and obese categories separately. As results were similar, all further analyses were run with the overweight and obese categories combined. Separate analyses were also run examining underweight adolescents separately, but as no differences were found, they were combined with the normal weight adolescents for all analyses. The exposure variables, factor scores for identified patterns in quintiles, were run as continuous variables. SES and MVPA were also run as continuous variables, whereas race/ethnicity was run as a categorical variable, with 'white' adolescents used as the reference group.

\section{Results}

\section{Cross-sectional associations}

Cross-sectional associations between factor scores for Time 1 dietary patterns and Time 1 weight status for each age and sex subgroup are shown in Table 2. The factor scores for each dietary pattern represent the level of adherence to that specific pattern, with higher factor scores representing a higher consumption of foods represented by that dietary pattern. At Time 1, higher scores for a 'vegetable' pattern were significantly associated with lower odds of being overweight/obese in older girls, but not in the other age and sex subgroups. In contrast to the study hypotheses, higher scores for a 'sweet \& salty snack food' pattern were associated with lower odds of being overweight/obese in the younger and older boys, and higher scores for a 'fruit' pattern were associated with higher odds of being overweight/obese in younger boys. These counterintuitive results were not seen in the older or younger girls. In fact, no significant associations were seen in the younger girl subgroup in Time 1 crosssectional analyses.

Cross-sectional associations between Time 2 dietary patterns and Time 2 weight status for each age and sex subgroup are shown in Table 3. At Time 2, higher scores for a 'vegetable \& fruit' pattern were associated with lower odds of being overweight/obese in older girls, similar to what was seen at Time 1. At Time 2, higher scores for a 'vegetable' pattern were associated with lower odds of being overweight/obese in the younger girls. Thus, similar inverse associations between scores for a pattern characterised by vegetables and risk of being overweight/obese were seen in both age cohorts of girls when they were in high school. No significant associations were seen in the younger or older boys in models adjusting for physical activity. In models not adjusting for physical activity, higher scores on a 'vegetable \& fruit' pattern and a 'sweet \& salty snack food' pattern were associated with lower odds of being overweight/obese in the older boys.

\section{Prospective and longitudinal associations}

Prospective and longitudinal associations between Time 1 dietary patterns and Time 2 weight status for each age and sex subgroup are shown in Table 4. In models not adjusting 
Table 2. Cross-sectional associations between Time 1 dietary patterns and Time 1 weight status (Odds ratios and $95 \%$ confidence intervals)

\begin{tabular}{|c|c|c|c|c|c|c|c|c|}
\hline \multirow[b]{2}{*}{ Time 1 dietary patterns } & \multicolumn{2}{|c|}{ Older girls } & \multicolumn{2}{|c|}{ Older boys } & \multicolumn{2}{|c|}{ Younger girls } & \multicolumn{2}{|c|}{ Younger boys } \\
\hline & OR† & $95 \% \mathrm{Cl}$ & OR & $95 \% \mathrm{Cl}$ & OR & $95 \% \mathrm{Cl}$ & OR & $95 \% \mathrm{Cl}$ \\
\hline \multicolumn{9}{|l|}{ Model $1 \ddagger$} \\
\hline Vegetable & $0.83^{*}$ & $0.75,0.92$ & 1.09 & $0.99,1.20$ & 1.08 & $0.92,1.26$ & 0.97 & $0.84,1 \cdot 12$ \\
\hline Fruit & 1.04 & $0.94,1 \cdot 15$ & 0.91 & $0.83,1.01$ & 1.00 & $0 \cdot 86,1 \cdot 17$ & $1 \cdot 24^{\star}$ & $1.06,1.44$ \\
\hline Starchy food & 0.89 & $0.79,1.01$ & 1.02 & $0.91,1.14$ & 1.00 & $0.84,1.18$ & 0.99 & $0.85,1.17$ \\
\hline Sweet \& salty snack food & 0.92 & $0.83,1.02$ & $0.87^{\star}$ & $0.80,0.96$ & 0.98 & $0.85,1 \cdot 15$ & $0.83^{*}$ & $0.71,0.96$ \\
\hline \multicolumn{9}{|l|}{ Model $2 \S$} \\
\hline Vegetable & $0.83^{*}$ & $0.74,0.92$ & 1.09 & $0.99,1.20$ & 1.07 & $0.92,1.26$ & 0.96 & $0.83,1 \cdot 11$ \\
\hline Fruit & 1.07 & $0.97,1.19$ & 0.92 & $0.83,1.01$ & 1.01 & $0.87,1 \cdot 18$ & $1 \cdot 23^{\star}$ & $1.05,1.43$ \\
\hline Starchy food & 0.91 & $0.80,1.04$ & 1.05 & $0.94,1.17$ & 1.00 & $0.85,1.19$ & 1.01 & $0.85,1.19$ \\
\hline Sweet \& salty snack food & 0.92 & $0.83,1.02$ & $0.88^{*}$ & $0.80,0.97$ & 0.98 & $0.85,1.15$ & $0.82^{*}$ & $0.71,0.95$ \\
\hline
\end{tabular}

\section{* $P \leq 0.05$.}

$\dagger$ OR for being overweight/obese $v$. normal weight for a one quintile increase in dietary pattern factor score.

$\ddagger$ Model 1 adjusts for race/ethnicity and socioeconomic status (SES).

$\S$ Model 2 adjusts for race/ethnicity, SES and physical activity.

for baseline weight status, results were similar to the Time 1 cross-sectional analyses. Higher scores for a 'vegetable' pattern were associated with decreased odds of being overweight/obese in older girls, higher scores for a 'sweet \& salty snack food' pattern were associated with decreased odds of being overweight/obese in older boys and higher scores for a 'fruit' pattern were associated with increased odds of being overweight/obese in the younger boys. All of the aforementioned associations lost significance after adjustment for baseline weight status. The only significant association found after adjustment for baseline weight status was a decrease in the odds of being overweight/obese in younger boys who scored high on a 'starchy food' pattern. It should be noted that significant associations between the 'starchy food' pattern and weight status were not seen in any other cross-sectional or prospective analysis in any subgroup.

\section{Discussion}

The primary aim of the present study was to examine the relationship between adherence to dietary patterns and weight status in a diverse cohort of adolescents. In general, few significant associations were found. Study results differed by sex, and overall showed little support for the study hypotheses that higher adherence to dietary patterns characterised by vegetables and fruits would be associated with a lower risk of being overweight or obese, and that the opposite would be true for patterns characterised by snack foods and fast food. In fact, higher adherence to a 'sweet and salty snack food' pattern was found to be significantly associated with lower risk of overweight/obese weight status in older and younger boys. In addition, a significant association was also seen in the younger boys between higher adherence to a 'fruit' pattern and a higher risk of being overweight or obese. An interesting

Table 3. Cross-sectional associations between Time 2 dietary patterns and Time 2 weight status (Odds ratios and $95 \%$ confidence intervals)

\begin{tabular}{|c|c|c|c|c|c|c|c|c|c|}
\hline \multirow[b]{2}{*}{ Time 2 dietary patterns } & \multicolumn{2}{|c|}{ Older girls } & \multicolumn{2}{|c|}{ Older boys } & \multirow[b]{2}{*}{ Time 2 dietary patterns } & \multicolumn{2}{|c|}{ Younger girls } & \multicolumn{2}{|c|}{ Younger boys } \\
\hline & OR† & $95 \% \mathrm{Cl}$ & OR & $95 \% \mathrm{Cl}$ & & OR & $95 \% \mathrm{Cl}$ & OR & $95 \% \mathrm{Cl}$ \\
\hline Model $1 \ddagger$ & & & & & Model 1 & & & & \\
\hline Vegetable \& fruit & $0.77^{\star}$ & $0.64,0.93$ & $0.82^{*}$ & $0.67,0.99$ & Vegetable & $0.64^{*}$ & $0.46,0.90$ & 1.07 & $0.85,1.35$ \\
\hline Starchy food & 0.94 & $0.78,1.14$ & $1 \cdot 12$ & $0.93,1.35$ & Fruit & 0.89 & $0.70,1.12$ & 1.21 & $0.93,1.58$ \\
\hline Sweet \& salty snack food & 0.93 & $0.80,1.09$ & $0.80^{*}$ & $0.67,0.96$ & Sweet \& salty snack food & 0.82 & $0.65,1.03$ & 0.91 & $0.70,1.18$ \\
\hline \multirow[t]{2}{*}{ Fast food } & $1 \cdot 12$ & $0.94,1.13$ & 1.04 & $0.86,1.24$ & Starchy food & 0.96 & $0.73,1.28$ & - & - \\
\hline & & & & & Fast food & - & - & 1.02 & $0.76,1.36$ \\
\hline Model $2 \S$ & & & & & Model 2 & & & & \\
\hline Vegetable \& fruit & $0.78^{\star}$ & $0.65,0.94$ & 0.86 & $0.71,1.06$ & Vegetable & $0.64^{*}$ & $0.46,0.90$ & 1.07 & $0.84,1.35$ \\
\hline Starchy food & 0.94 & $0.78,1.14$ & $1 \cdot 16$ & $0.97,1.40$ & Fruit & 0.91 & $0.71,1.15$ & 1.25 & $0.95,1.63$ \\
\hline Sweet \& salty snack food & 0.93 & $0.80,1.09$ & 0.84 & $0.70,1.01$ & Sweet \& salty snack food & 0.83 & $0.66,1.04$ & 0.94 & $0.72,1.22$ \\
\hline \multirow[t]{2}{*}{ Fast food } & $1 \cdot 11$ & $0.94,1.31$ & 0.95 & $0.78,1.14$ & Starchy food & 1.01 & $0.76,1.35$ & - & - \\
\hline & & & & & Fast food & - & - & 0.98 & $0.73,1.32$ \\
\hline
\end{tabular}

${ }^{*} P \leq 0.05$.

†OR for being overweight/obese $v$. normal weight for a one quintile increase in dietary pattern factor score.

$\ddagger$ Model 1 adjusts for race/ethnicity and socioeconomic status (SES).

$\S$ Model 2 adjusts for race/ethnicity, SES and physical activity. 
Table 4. Prospective and longitudinal associations between Time 1 dietary patterns and Time 2 weight status

(Odds ratios and $95 \%$ confidence intervals)

\begin{tabular}{|c|c|c|c|c|c|c|c|c|}
\hline \multirow[b]{2}{*}{ Time 1 dietary patterns } & \multicolumn{2}{|c|}{ Older girls } & \multicolumn{2}{|c|}{ Older boys } & \multicolumn{2}{|c|}{ Younger girls } & \multicolumn{2}{|c|}{ Younger boys } \\
\hline & OR† & $95 \% \mathrm{Cl}$ & OR & $95 \% \mathrm{Cl}$ & OR & $95 \% \mathrm{Cl}$ & OR & $95 \% \mathrm{Cl}$ \\
\hline \multicolumn{9}{|l|}{ Model $1 \ddagger$} \\
\hline Vegetable & $0.85^{*}$ & $0.75,0.97$ & $1 \cdot 11$ & $0.97,1.28$ & 0.97 & $0.80,1.18$ & $1 \cdot 11$ & $0.88,1.41$ \\
\hline Fruit & 1.00 & $0.88,1 \cdot 13$ & 0.98 & $0.85,1.13$ & 1.02 & $0.83,1.25$ & $1.47^{\star}$ & $1.13,1.91$ \\
\hline Starchy food & 0.94 & $0.81,1.09$ & 0.96 & $0.82,1.13$ & 0.96 & $0.78,1.19$ & 0.78 & $0.60,1.01$ \\
\hline Sweet \& salty snack food & 0.93 & $0.82,1.05$ & $0.86^{\star}$ & $0.74,0.98$ & 0.89 & $0.73,1.08$ & 0.84 & $0.66,1.06$ \\
\hline \multicolumn{9}{|l|}{ Model $2 \S$} \\
\hline Vegetable & $0.85^{*}$ & $0.75,0.97$ & $1 \cdot 11$ & $0.96,1.27$ & 0.97 & $0.70,1.18$ & 1.09 & $0.86,1.39$ \\
\hline Fruit & 1.02 & $0.90,1.15$ & 0.98 & $0.85,1.13$ & 1.02 & $0.83,1.25$ & $1.47^{*}$ & $1.13,1.92$ \\
\hline Starchy food & 0.94 & $0.81,1.09$ & 0.98 & $0.83,1.15$ & 0.96 & $0.78,1.19$ & 0.79 & $0.60,1.04$ \\
\hline Sweet \& salty snack food & 0.93 & $0.82,1.05$ & $0.85^{\star}$ & $0.74,0.98$ & 0.89 & $0.73,1.08$ & 0.85 & $0.67,1.08$ \\
\hline \multicolumn{9}{|l|}{ Model 3q } \\
\hline Vegetable & 0.87 & $0.74,1.03$ & 1.08 & $0.91,1.29$ & 0.96 & $0.75,1.24$ & 1.23 & $0.92,1.65$ \\
\hline Fruit & 0.94 & $0.80,1 \cdot 10$ & 1.03 & $0.86,1.22$ & 1.00 & $0.76,1.31$ & 1.27 & $0.92,1.74$ \\
\hline Starchy food & 0.93 & $0.77,1 \cdot 14$ & 0.98 & $0.81,1.20$ & 0.98 & $0.74,1.28$ & $0.70^{*}$ & $0.50,0.98$ \\
\hline Sweet \& salty snack food & 0.97 & $0.83,1.14$ & 0.86 & $0.72,1.03$ & 0.92 & $0.71,1.19$ & 0.99 & $0.74,1.34$ \\
\hline
\end{tabular}

* $P \leq 0.05$.

† OR for being overweight/obese $v$. normal weight for a one quintile increase in dietary pattern factor score.

$\ddagger$ Model 1 adjusts for race/ethnicity and socioeconomic status (SES).

$\S$ Model 2 adjusts for race/ethnicity, SES and physical activity.

I Model 3 adjusts for race/ethnicity, SES, physical activity, and Time 1 weight status.

finding was that all age and sex subgroups showed at least a marginal inverse association between a 'sweet \& salty snack food' pattern and risk for overweight/obese weight status in prospective analyses not adjusting for baseline weight status. Thus, there was no hint that adherence to this pattern characterised by snack foods might increase risk of overweight or obesity in any of the age and sex subgroups. For the 'fast food' pattern, we found no indication of an association in any direction between adherence to this pattern and weight status. Therefore, the present results in no way supported our hypothesis that a higher adherence to dietary patterns characterised by snack foods or fast food would be associated with higher risk of overweight/obese weight status.

The only results that aligned with the study hypotheses were the inverse associations found between patterns loading heavily on vegetables and risk of overweight/obese weight status for the older and younger girls. The inverse relationship between a 'vegetable' pattern and risk for overweight/obesity in the younger girls was seen only at Time 2 . Thus, an inverse relationship between a 'vegetable' pattern was seen in both older and younger girls while they were in high school, and the fact that this relationship was also seen in older girls at Time 2 (when they were post-high school) indicates that this relationship may continue in girls as they grow older.

Little research has been done examining the relationship between dietary patterns and weight, and the majority of studies have also found inconsistent results. Ritchie et al. ${ }^{(9)}$ examined the relationship between dietary patterns and weight in adolescents in the USA, and they used cluster analysis to identify patterns from dietary data collected over 10 years in black and white adolescent females (aged 9-11 years at baseline). A 'healthy' pattern was identified, characterised by a high intake of fruits, vegetables, dairy, grains without added fats, mixed dishes and soups, and a low intake of sweetened drinks, other sweets, fried foods, burgers and pizza $^{(9)}$. Similar to the present results, this 'healthy' pattern was significantly related to a smaller mean increase in waist circumference over time in white females ${ }^{(9)}$.

The relationship between dietary patterns and weight in adolescent populations outside the USA has been examined by four other studies ${ }^{(10-13)}$. McNaughton et al. ${ }^{(11)}$ identified dietary patterns in Australian adolescents aged 12-18 years using factor analysis. Similar to the present results, high consumers of a 'high fat and sugar' pattern, characterised by high intake of meat pies, sausage rolls or other savoury pastries, cream, chocolate biscuits, chocolate, sweet pies or sweet pastries, potato chips, corn chips, hamburgers, pizza and regular soft drinks, tended to have a lower BMI, although these results were not statistically significant ${ }^{(11)}$. Okubo et al. ${ }^{(10)}$ examined dietary patterns in a cohort of late adolescent females (aged 18-20 years) in Japan using factor analysis and identified a 'healthy' pattern characterised by a high intake of green and white vegetables, mushrooms, seaweeds, potatoes, fish and shellfish, soya products, processed fish, fruit and salted vegetables. Similar to the present results, they found that this 'healthy' pattern was significantly associated with a lower risk of obesity in females. They also found that a 'Japanese traditional' pattern, characterised by high intake of rice, miso soup and soya products and a 'Western' pattern characterised by meats, fats and oils, seasonings and processed meats and eggs, were significantly associated with an higher risk of obesity $^{(10)}$. Song et al. ${ }^{(12)}$ identified dietary patterns in a cohort of Korean adolescents, and they found that higher scores on a 'Western' pattern, characterised by a high intake of starch and bread, pizza and hamburgers, meat and meat products, dairy products and soft drinks, were associated with higher risk of being overweight in boys, whereas no significant associations were found in girls. Craig et al. ${ }^{(13)}$ 
identified dietary patterns in a cohort of Scottish adolescents aged 12-17 years and found no consistent association between the identified patterns and BMI in the adolescent group, although a result similar to ours was found in the child group (aged 5-11 years). They identified a 'snacks' pattern in this age group, characterised by high intake of foods including chocolate bars, wrapped ice cream, other ice cream, chocolate biscuits, regular crisps, other savoury snacks, sausages, meat burgers and regular fizzy drinks ${ }^{(13)}$. The lowest scores for the 'snacks' pattern were found in obese children (i.e. the obese children reported eating less of the foods loading heavily on the 'snacks' pattern) ${ }^{(13)}$.

We do not know exactly why such inconsistent associations between dietary pattern adherence and weight status were found in the present study and others in adolescent populations, but there are a few possible explanations. We hypothesised that adhering highly to a pattern characterised by high-energy foods would be associated with obesity as we believed these individuals would consume more energy, but this may not be the case. A FFQ was used to assess usual diet, and as with any study using a FFQ, underreporting of usual intake or invalid reporting because of social desirability bias is possible. Additionally, a semi-quantitative FFQ was used in the present study, and although this FFQ did assess portion sizes for many foods, it did not assess portion sizes for all foods. Two adolescents consuming a food at the same frequency could be taking in different amounts of energy because of differences in portion size. In addition, diet may not be the primary factor in determining weight status in this age group. We hypothesise that poor dietary intake may not have the impact on weight status in adolescents as it does in adult populations, making it that much more difficult to find consistent associations between diet and obesity in youth.

Strengths of the present study include a large, ethnically and socioeconomically diverse population with data over two time points that allowed us to examine longitudinal and secular changes over a 5-year period. Several limitations of the present study must be considered. A FFQ was used to assess usual diet, and as with any assessment of dietary intake, underreporting of usual intake or invalid reporting because of social desirability bias is possible. In addition, although the FFQ was administered by trained staff at Time 1, it was self-administered at Time 2 . The outcome variable, weight status, was based on BMI, which was calculated from selfreported height and weight variables at both Time 1 and Time 2. Although it would have been preferable to have had measured height and weight, the correlations between observed and self-reported BMI at Time 1 were high, which lessens concerns over using self-reported $\mathrm{BMI}^{(15)}$. In addition, cross-sectional analyses were run at Time 1 using both measured and self-reported BMI and results were similar (results not shown). We were limited to examining BMI as no other obesity index was measured in Project EAT (i.e. body fat, lean body mass or waist circumference), and we cannot say whether the dietary patterns identified in the present study may have been associated with other measures of obesity. For weight status, we used the Must et al. ${ }^{(16)}$ classification. Although this can complicate comparisons between the present study and others using reference values from the International Obesity Task Force ${ }^{(25)}$, after rerunning analyses using the International Obesity Task Force reference values, the present study conclusions did not change. Although we did a longitudinal analysis, it is not possible to infer causality. We do not know for certain that adherence to dietary patterns did not change over the 5-year period. As with any observational analysis, unmeasured or unexamined variables may partly explain the outcome. Finally, although an examination of dietary patterns has certain advantages over traditional methods of examining single foods or nutrients, results can be more challenging to interpret.

In conclusion, we did not find consistent or intuitive associations between dietary patterns identified through principal components factor analysis and weight status in the Project EAT cohort of adolescents. The identified dietary patterns may not capture the elements of diet that are truly important in determining adolescent weight, or diet may not be the primary driver in determining weight status at this age. Dietary pattern analysis methods have rarely been used in adolescent populations, but offer a useful way to examine the relationship between dietary intake and obesity in this age group. Further research is warranted using different dietary pattern analysis methods in adolescent populations, including reduced rank regression. Using a variety of methods will probably provide the most information on the relationship between dietary patterns and weight status in adolescents.

\section{Acknowledgements}

The present study was supported by Grant R40 MC 00319 (PI: Neumark-Sztainer) from the Maternal and Child Health Bureau (Title V Social Security Act), Health Resources and Service Administration, Department of Health and Human Services. G. J. C. was supported, in part, by the Adolescent Health Protection Program (School of Nursing, University of Minnesota) grant number T01-DP000112 (PI: Bearinger) from the Centers for Disease Control and Prevention (CDC). The contents of the present paper are solely the responsibility of the authors and do not necessarily represent the official views of the CDC. G. J. C., A. F., P. J. H. and D. N.-S. were involved in study design; G. J. C. analysed data and prepared the manuscript; A. F., P. J. H., J. L. S. and D. N.-S. gave input on statistical analysis, drafting of the manuscript and interpretation of the results; G. J. C. had primary responsibility for final content. All authors read and approved the final manuscript. The authors declare no conflict of interest.

\section{References}

1. Ogden CL, Carroll MD, Curtin LR, et al. (2006) Prevalence of overweight and obesity in the United States, 1999-2004. JAMA 295, 1549-1555.

2. Newby PK (2007) Are dietary intakes and eating behaviors related to childhood obesity? A comprehensive review of the evidence. J Law Med Ethics 35, 35-60.

3. Newby PK (2009) Plant foods and plant-based diets: protective against childhood obesity? Am J Clin Nutr 89, 1572 S-1587S 
4. Togo P, Osler M, Sorensen TI, et al. (2001) Food intake patterns and body mass index in observational studies. Int J Obes Relat Metab Disord 25, 1741-1751.

5. Newby PK \& Tucker KL (2004) Empirically derived eating patterns using factor or cluster analysis: a review. Nutr Rev 62, 177-203.

6. Tucker KL (2010) Dietary patterns, approaches, and multicultural perspective. Appl Physiol Nutr Metab 35, 211-218.

7. Marchioni DM, Latorre Mdo R, Eluf-Neto J, et al. (2005) Identification of dietary patterns using factor analysis in an epidemiological study in Sao Paulo. Sao Paulo Med J 123, $124-127$

8. van Dam RM (2005) New approaches to the study of dietary patterns. Br J Nutr 93, 573-574.

9. Ritchie LD, Spector P, Stevens MJ, et al. (2007) Dietary patterns in adolescence are related to adiposity in young adulthood in black and white females. J Nutr 137, 399-406.

10. Okubo H, Sasaki S, Murakami K, et al. (2008) Three major dietary patterns are all independently related to the risk of obesity among 3760 Japanese women aged 18-20 years Int J Obes (Lond) 32, 541-549.

11. McNaughton SA, Ball K, Mishra GD, et al. (2008) Dietary patterns of adolescents and risk of obesity and hypertension. I Nutr 138, 364-370.

12. Song Y, Park MJ, Paik HY, et al. (2010) Secular trends in dietary patterns and obesity-related risk factors in Korean adolescents aged 10-19 years. Int J Obes (Lond) 34, 48-56.

13. Craig LC, McNeill G, Macdiarmid JI, et al. (2010) Dietary patterns of school-age children in Scotland: association with socio-economic indicators, physical activity and obesity. Br J Nutr 103, 319-334.

14. Cutler GJ, Flood A, Hannan P, et al. (2009) Major patterns of dietary intake in adolescents and their stability over time. J Nutr 139, 323-328.

15. Himes JH, Hannan P, Wall M, et al. (2005) Factors associated with errors in self-reports of stature, weight, and body mass index in Minnesota adolescents. Ann Epidemiol 15, $272-278$

16. Must A, Dallal GE \& Dietz WH (1991) Reference data for obesity: 85th and 95th percentiles of body mass index (wt/ht2) and triceps skinfold thickness. Am J Clin Nutr $\mathbf{5 3}$ $839-846$.

17. Rockett HR, Breitenbach M, Frazier AL, et al. (1997) Validation of a youth/adolescent food frequency questionnaire. Prev Med 26, 808-816.

18. Rockett HR, Wolf AM \& Colditz GA (1995) Development and reproducibility of a food frequency questionnaire to assess diets of older children and adolescents. I Am Diet Assoc 95, 336-340.

19. Rockett HR, Berkey CS \& Colditz GA (2003) Evaluation of dietary assessment instruments in adolescents. Curr Opin Clin Nutr Metab Care 6, 557-562.

20. Cullen KW \& Zakeri I (2004) The youth/adolescent questionnaire has low validity and modest reliability among low-income African-American and Hispanic seventh- and eighth-grade youth. J Am Diet Assoc 104, 1415-1419.

21. Kristal AR, Feng Z, Coates RJ, et al. (1997) Associations of race/ethnicity, education, and dietary intervention with the validity and reliability of a food frequency questionnaire: the Women's Health Trial Feasibility Study in Minority Populations. Am J Epidemiol 146, 856-869.

22. Utter J, Neumark-Sztainer D, Jeffery R, et al. (2003) Couch potatoes or french fries: are sedentary behaviors associated with body mass index, physical activity, and dietary behaviors among adolescents? I Am Diet Assoc 103, 1298-1305.

23. Godin G \& Shephard RJ (1985) A simple method to assess exercise behavior in the community. Can J Appl Sport Sci 10, 141-146.

24. Sallis JF, Buono MJ, Roby JJ, et al. (1993) Seven-day recall and other physical activity self-reports in children and adolescents. Med Sci Sports Exerc 25, 99-108.

25. Cole TJ, Bellizzi MC, Flegal KM, et al. (2000) Establishing a standard definition for child overweight and obesity worldwide: international survey. BMJ 320, 1240-1243.

26. Neumark-Sztainer D, Story M, Hannan PJ, et al. (2002) Overweight status and eating patterns among adolescents: where do youths stand in comparison with the healthy people 2010 objectives? Am J Public Health 92, 844-851. 